\title{
Erratum to: CBP binding outside of promoters and enhancers in Drosophila melanogaster
}

Philge Philip ${ }^{1,2,3}$, Ann Boija ${ }^{4}$, Roshan Vaid ${ }^{4}$, Allison M. Churcher ${ }^{1}$, David J. Meyers ${ }^{5}$, Philip A. Cole ${ }^{5}$, Mattias Mannervik ${ }^{4^{*}}$ and Per Stenberg ${ }^{1,2,6^{*}}$

\section{Erratum to: Epigenetics \& Chromatin (2015) 8:48 DOI 10.1186/s13072-015-0042-4}

After the publication of this work [1], it was noticed that the funding source was inadvertently omitted from the acknowledgements. Please see the corrected acknowledgements statement.

\begin{abstract}
Author details
1 Department of Molecular Biology, Umea Umeå University, 90187 Umeå, Sweden. ${ }^{2}$ Computational Life Science Cluster (CLiC), Umeå University, 901 87 Umeå, Sweden. ${ }^{3}$ Centre for Cellular and Molecular Biology, Uppal Road, Hyderabad, Telangana 500007, India. ${ }^{4}$ Department of Molecular Biosciences, The Wenner-Gren Institute, Stockholm University, 10691 Stockholm, Sweden.

${ }^{5}$ Department Pharmacology and Molecular Sciences, The Johns Hopkins University School of Medicine, 725 North Wolfe Street, Baltimore, MD 21205, USA. ${ }^{6}$ Division of CBRN Security and Defence, FOI-Swedish Defence Research Agency, Umeå, Sweden.
\end{abstract}

\section{Acknowledgements}

This work was supported by grants from the Knut and Alice Wallenberg (to EpiCoN, co-PI: PS), Kempe, Åke Wiberg, Magnus Bergvall and Carl Trygger foundations to Per Stenberg and the Swedish Cancer Foundation (Cancerfonden) to Mattias Mannervik. We thank the FAMRI (Flight Attendant Medical Research Institute) Foundation for support of this work.

The online version of the original article can be found under doi:10.1186/s13072-015-0042-4.

Received: 1 September 2016 Accepted: 1 September 2016 Published online: 12 September 2016

\author{
Reference \\ 1. Philip P, Boija A, Vaid R, Churcher AM, Meyers DJ, Cole PA, et al. CBP bind- \\ ing outside of promoters and enhancers in Drosophila melanogaster. \\ Epigenetics Chromatin. 2015;8:48.
}

*Correspondence: mattias.mannervik@su.se; per.stenberg@umu.se ${ }^{4}$ Department of Molecular Biosciences, The Wenner-Gren Institute, Stockholm University, 10691 Stockholm, Sweden

${ }^{6}$ Division of CBRN Security and Defence, FOI-Swedish Defence Research Agency, Umeå, Sweden

Full list of author information is available at the end of the article 